\title{
Robust Fuzzy-Second Order Sliding Mode based Direct Power Control for Voltage Source Converter
}

\author{
D. Kairous and B. Belmadani \\ Laboratoire de Génie électrique et Energie Renouvelable -LGEER-. Departement of Electrical Engeneering. \\ Faculty of Technology. University of Hassiba Ben-Bouali at Chlef -UHBC-.
}

Chlef. Algeria

\begin{abstract}
This paper focuses on a second order sliding mode based direct power controller (SOSM-DPC) of a three-phase grid-connected voltage source converter (VSC). The proposed control scheme combined with fuzzy logic aims at regulating the DC-link voltage of the converter and precisely tracking arbitrary power references, in order to easily control the system's power factor. Therefore measures are proposed to reduce the chattering effects inherent to sliding-mode control (SMC). Simulations performed under Matlab/Simulink validate the feasibility of the designed Fuzzy-SOSM. Simulation results on a 1kVA gridconnected VSC under normal and faulted grid voltage conditions demonstrate good performance of the proposed control law in terms of robustness, stability and precision.
\end{abstract}

Keywords-AC-DC power converters; Bidirectional power flow; Fuzzy logic; Sliding mode control; Direct Power control

\section{INTRODUCTION}

Voltage source converter (VSC) is a very useful device. Among many desirable characteristics [1], the VSC permit the independent control of active and reactive power, what makes it very attractive for power conditioning and transmission. As such, the VSC is a key element in power electronics-based equipment for flexible AC transmission systems (FACTS), high-voltage direct current (HVDC) systems and active power filters (APFs). Furthermore, with the development of smart grids involving distributed generation and the interconnection of renewable power generation systems [2], such as wind farms, photovoltaic, solar thermal plants, etc., the need for power flow steering increases. In this context, efficient control strategies for grid connected VSCs are relevant more than ever.

PI control is a mature and proven method which is very popular for industrial applications. Effective regulation of the active and reactive power flows between the grid and the VSC can be achieved with PI-based vector control. However, in order to apply PI regulation techniques at vector control, feedforward compensation must be employed to eliminate crosscoupling and linearize the system's model. Owing to this linearization, the control scheme and the performance of vector control become respectively very sensitive to the system's parameters and dependent on their accuracy [3].

Sliding-mode control (SMC) is a variable structure control strategy that uses a special version of on-off control, or high frequency switching, to achieve robust control of non-linear systems [4-5]. A good mathematical background for SMC is presented in [6], along with a demonstration of its applicability to electric drives.

Sliding mode control is based on the theory of the variable-structure systems (VSS). In a closed loop system, one way to change the structure is to use different controllers depending on the state of the system. The main idea is to switch rapidly between strong control actions when the system deviates from the desired response. To do this, the closed-loop system behavior must be described by a switching surface in the state space. Then, the control actions can be chosen so that the net effect of the switching (chattering) will be to move the system towards the switching surface [4]. When this is achieved, the system will "slide" along the switching surface, giving what one calls the "sliding-mode". A regain of interest in SMC occurred in the 1980s with the increasing availability of powerful microprocessors. The robustness of the method then began to be recognized along with its relative immunity toward external disturbances and its low sensitivity to system parameters variations [5]. In this work, a second order sliding mode (SOSM) control scheme is proposed, to ensure tracking of the DC bus voltage and rotor power factor in a wind power systems. The control technique named super-twisting is based on a bounded continuous control with discontinuities in the control derivative [5].

In objective to attenuating the chattering effect the proposed control will be associated with fuzzy logic [7]. In fact, during the last decade, the fuzzy logic control (FLC) has been selected as suitable control solution in the field of power electronics and drives [8]. Among the advantages provided by this control approach over the conventional controllers in other hand it does not require accurate mathematical model. It can thus work with inaccurate inputs, handle nonlinear model systems and easily reach performances of ideal digital PI controllers. On the other hand, The SMC appears as a simple way to design robust controllers for electrical drives, a powerful technique to eliminate sensors in electrical machine drives. Furthermore, the SMC does not require many computational operations and remains insensitive to plant parameters variations. Accordingly, this paper aims at combining the advantages of FLC and SMC for robust control electronic power converter. This approach has been successful applied in wide area.

The rest of this paper is organized as follows. In section II, the global wind energy conversion system (WECS) is described. Section III then presents the model of the VSC to be controlled. In section IV and V, the details of the sliding mode control law and fuzzy logic are presented respectively. 
Then, the section VI described the used space vector SVPWM. Numerical simulations results and analysis for both transient and steady state are presented in section $\mathrm{V}$ and conclusions are drawn in section VI.

\section{EXCHANGE POWERS BY GRID SIDE CONVERTER}

This work addresses the usual scheme adopted for the doubly fed induction generator (DFIG) in a variable speed, constant frequency wind power generation system. Imposing slip frequency, amplitude and phase on the rotor voltage permits to achieve constant frequency, constant voltage output at the stator.

The structure of this system is depicted on Fig. 1. The rotor is connected to grid via two voltage-source PWM converters, connected back-to-back: the rotor-side converter (RSC) and the grid-side converter (GSC) [9].

As the Fig.1 suggests, the two converters do have the same circuit structure. They simply alternate between rectifier and

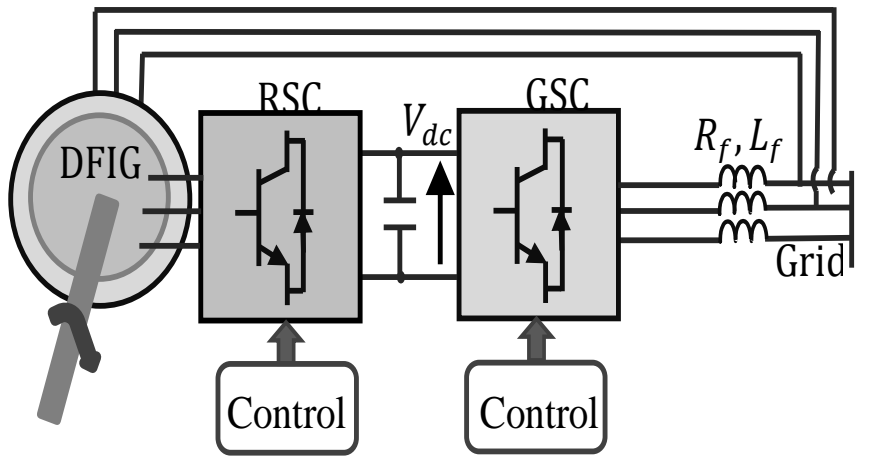

Fig. 1. Bloc diagram of WECS connected to the grid

inverter function, depending on the flowing direction of rotor energy.

The RSC feeds excitation current into rotor winding and achieves flux orientation, to catch maximum wind energy and adjust reactive power output. Depending on the rotor speed relative to the generator synchronous speed, the RSC works as either an inverter (low speed) or a rectifier (high speed). When the generator is working at synchronous speed, the RSC feeds direct current excitation into rotor, working as a Chopper. The GSC works in dual cooperation with the RSC, permitting seamless energy flow in both directions. It also controls DC bus voltage and adjust grid-side power factor, which makes the entire wind power system to have a flexible reactive power regulation.

\section{SYSTEM MODEL}

The GSC system can be modeled as an ideal VSC. The equivalent circuit of Fig. 2 is a simplified representation of the grid-connected VSC in the stationary $\alpha \beta$ reference frame.

KVL applied to the equivalent circuit of Fig. 2 gives the relation between line current and the supply voltage as [1]

$$
U_{\alpha \beta}=I_{\alpha \beta} R_{f}+L_{f} \frac{d I_{\alpha \beta}}{d t}+V_{\alpha \beta}
$$

From (1), the derivative of the current can be written as

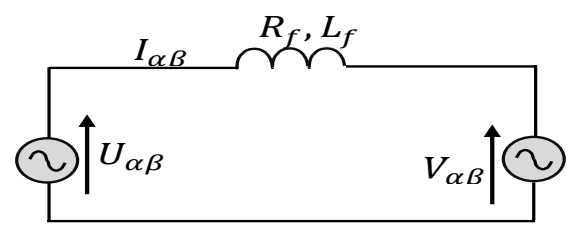

Fig. 2. Equivalent circuit of VSC in stationary frame

$$
\frac{d I_{\alpha \beta}}{d t}=\left(1 / L_{f}\right)\left(U_{\alpha \beta}-R_{f} I_{\alpha \beta}-V_{\alpha \beta}\right)
$$

The power exchange with the electric grid, as seen from the network side (in stationary frame), is defined by (3)

$$
\begin{aligned}
& P=(-3 / 2)\left(U_{\alpha} I_{\alpha}+U_{\beta} I_{\beta}\right) \\
& Q=(-3 / 2)\left(U_{\beta} I_{\alpha}-U_{\alpha} I_{\beta}\right)
\end{aligned}
$$

From (3), the derivative of the current may be written as

$$
\left[\begin{array}{l}
d I_{\alpha} / d t \\
d I_{\beta} / d t
\end{array}\right]=\left(\frac{-2}{3 U^{2}}\right) \frac{d}{d t}\left\{\left[\begin{array}{cc}
-U_{\alpha} & -U_{\beta} \\
-U_{\beta} & U_{\alpha}
\end{array}\right]\left[\begin{array}{l}
P \\
Q
\end{array}\right]\right\}
$$

Knowing that the derivative of the grid voltage, expressed in the stationary reference frame, is

$$
\begin{aligned}
& d U_{\alpha} / d t=w U \cos (w t)=-w U_{\beta} \\
& d U_{\beta} / d t=w U \sin (w t)=w U_{\alpha}
\end{aligned}
$$

Equating relations (2) and (4) gives an expression for the derivatives of the powers as

$$
\begin{gathered}
d P / d t=\left(-3 / 2 L_{f}\right)\left[\left(U_{\alpha}^{2}+U_{\beta}^{2}\right)-\left(U_{\alpha} V_{\alpha}+U_{\beta} V_{\beta}\right)\right] \\
-\left(R_{f} / L_{f}\right) P-w Q \\
d Q / d t=\left(-3 / 2 L_{f}\right)\left[-\left(U_{\beta} V_{\alpha}+U_{\alpha} V_{\beta}\right)\right]-\left(R_{f} / L_{f}\right) Q \\
+w P
\end{gathered}
$$

This last expression relating the time derivative of the powers to the voltage is essential to the design of the SMC below.

\section{Controller Design}

The control problem can be stated as: "find the sequence and the duration of the on-off states for converter switches so that the given dynamical specifications of the closed-loop system are satisfied".

This formulation is a good starting point for introducing a sliding-mode control since it intimately links the selection of the PWM switching pattern to the dynamics of the system and will result in a PWM pattern that directly forces the close-loop behavior of the converter to slide along the desired switching surface.

The first design step consists in describing a suitable switching surface along which the system will be allowed to slide. Then one must define the reaching law that will force the system to move towards this very specific surface. Finally, an adequate technique should be introduced to alleviate the side effects of the chattering of the command on the switching surface [6]. 


\section{A. Switching surface}

The switching surface can be designed to ensure that the power $P$ and $Q$ track their references $P^{*}$ and $Q^{*}$. The description of such a surface can be obtained by taking the errors on the actual values of the powers as [10].

$$
\begin{aligned}
& e_{P}(t)=P^{*}-P \\
& e_{Q}(t)=Q^{*}-Q
\end{aligned}
$$

Since the desired motion of the system will be obtained for $e(t)=0$, the switching surface can be defined in terms of these errors as

$$
\begin{aligned}
& S_{P}=e_{P}(t)+K_{P} \int e_{P}(\tau) d \tau-e_{P}(0) \\
& S_{Q}=e_{Q}(t)+K_{Q} \int e_{Q}(\tau) d \tau-e_{Q}(0)
\end{aligned}
$$

Where $K_{P}$ and $K_{Q}$ are positive control gains.

Whenever the system reaches the switching surface and slides along it, we have:

$$
S_{P}=S_{Q}=d S_{P} / d t=d S_{Q} / d t=0
$$

According to (8), when the derivatives of $S_{P}$ and $S_{Q}$ are zero, we get the derivative for the errors as

$$
\begin{aligned}
& d e_{P}(t) / d t=-K_{P} e_{P}(t) \\
& d e_{Q}(t) / d t=-K_{Q} e_{Q}(t)
\end{aligned}
$$

The solution $\mathrm{a}=\mathrm{of}(10)$ are decreasing exponentials, which ensure that the power errors converge asymptotically to zero with the time constants of $1 / K_{P}$ and $1 / K_{Q}$.

\section{B. Sliding Mode based Direct Power Control (SM-DPC) Law}

The second step focus on the synthesis of an appropriate control law to force the state trajectories to slide along the switching surface. Taking the time derivative of (8), we get

$$
\left[\begin{array}{l}
d S_{P} / d t \\
d S_{Q} / d t
\end{array}\right]=\left[\begin{array}{l}
F_{P} \\
F_{Q}
\end{array}\right]+D\left[\begin{array}{l}
V_{\alpha} \\
V_{\beta}
\end{array}\right]=0
$$

This gives the equivalent control voltages

$$
\left[\begin{array}{l}
V_{\alpha} \\
V_{\beta}
\end{array}\right]=\left[\begin{array}{l}
V_{P e q} \\
V_{Q e q}
\end{array}\right]=-D^{-1}\left[\begin{array}{c}
F_{P} \\
F_{Q}
\end{array}\right]
$$

Where, according to (6) and (7)

$$
\begin{gathered}
F_{P}=\left(3 / 2 L_{f}\right)\left(U_{\alpha}^{2}+U_{\beta}^{2}\right)+ \\
\left.+R_{f} / L_{f}\right) P+w Q \\
+K_{P}\left(P^{*}-P\right) \\
F_{Q}=\left(R_{f} / L_{f}\right) Q-w Q+K_{Q}\left(Q^{*}-Q\right)
\end{gathered}
$$

And

$$
-D^{-1}=\left(2 L_{f} / 3 U^{2}\right)\left[\begin{array}{cc}
U_{\alpha} & U_{\beta} \\
U_{\beta} & -U_{\alpha}
\end{array}\right]
$$

Relation (12) above is the control law for the power controller. The control voltages are directly the converter's output voltages in the stationary reference frame, which are used to generate the PWM switching scheme that will keep the system on the switching surface.

In sliding-mode control design, one must derive the conditions under which the control law will stabilize the system by driving its state trajectory to an equilibrium surface with good robustness. We use the quadratic Lyapunov function

$$
W=0.5 S^{T} S \geq 0
$$

The time derivate of which can be expressed in terms of the state trajectories (12)

$$
d W / d t=d\left(0.5 S^{T} S\right) / d t=S^{T} d S / d t=S^{T}(F+D V)
$$

In order to guaranty stability, the control law must thus be modified so that the time derivative $d W / d t$ is definite negative when $S \neq 0$. This condition can be achieved with the control law

$$
\left[\begin{array}{l}
V_{\alpha} \\
V_{\beta}
\end{array}\right]=-D^{-1}\left(\left[\begin{array}{l}
F_{P} \\
F_{Q}
\end{array}\right]+\left[\begin{array}{cc}
K_{\operatorname{sgn} P} & 0 \\
0 & K_{\operatorname{sgn} Q}
\end{array}\right]\left[\begin{array}{l}
\operatorname{Sgn}\left(S_{P}\right) \\
\operatorname{Sgn}\left(S_{Q}\right)
\end{array}\right]\right)
$$

where $K_{s g n P}$ and $K_{s g n Q}$ are positive control gains, while $\operatorname{Stgn}\left(S_{P}\right)$ and $\operatorname{Sgn}\left(S_{Q}\right)$ are sign functions. By setting appropriate constant values, stability can be achieved.

\section{Power chattering attenuation}

Because the SM involve fast switching of the command, unexpected chattering of the command may result in undesirable behavior of the system, e.g. excite some high frequency mode of the system generating instability.

One way to alleviate this problem is to modify the sign function in equation (17) to introduce a boundary layer that smooth the command around the sliding surface. Such a modification in the surface neighborhood can be written

$$
\operatorname{Sgn}\left(S_{i}\right)= \begin{cases}1 & \text { if } S_{i}>K_{\mathrm{sgn} i} \\ S_{i} / \lambda_{\mathrm{sgn} i} & \text { if }\left|S_{i}\right|>K_{\mathrm{sgn} i} \\ -1 & \text { if } S_{i}<K_{\mathrm{sgn} i}\end{cases}
$$

Where $i$ stands for $P$ or $Q$ and $\lambda_{\mathrm{sgn} i}$ is the width of the boundary layer.

To ensure an even more robust tracking of the powers exchanged by the converter, we apply the super-twisting algorithm (STA), by adding a new term $V_{i S T}$ to the control law $V_{i e q}$ given by (17).

The sliding along the surface (9) can be obtained by applying only the new law

$$
V_{i}=V_{i S T}+V_{i e q}
$$

The new term $V_{i S T}$ is added to the equivalent control law, based on the SOSM (STA approach) [10] [11].

The idea of the SOSM control techniques is to zero a function of the system's states, the sliding variable $S$, and its

first time derivative $S$. The function is designed based on the desired control objectives, to guarantee their achievement when $S=0$.

The condition of stabilizing $S=\dot{S}=0$ determines the 2sliding manifold in the state space.

The SOSM algorithm acts taking the trajectories in the state space to the 2-sliding manifold in finite time and keeping 
them operating robustly on it, i.e., makes the system operate in SOSM.

Discontinuous control input of the SOMC directly influences the sign and the magnitude of the second order time derivative of the sliding manifold. Since its control structure is relatively simple and no much information is needed, it has become the most widely used high order sliding mode control method [11].

The main problem with high-order sliding mode algorithm implementations is the increased required information. Indeed, the implementation of an nth-order controller requires the knowledge of $\dot{S}, \ddot{S}, \ldots, \stackrel{(n-1)}{S}$

There are several SOSM algorithms, each of them with their own characteristics. In particular, the super-twisting algorithm has a quite simple law and allows synthesizing a continuous control action with discontinuous time derivative (in contradiction to those of the sub-optimal algorithm). and it only requires measurements of surface $s$.

The control law in equation (19) is composed of switching control terms and equivalent control terms. Where $V_{i S T}$ are the switching control terms. The switching control terms make the system in any initial state reach the sliding manifold in finite time, which are calculated through application of the super-twisting algorithm in this paper.

$V_{i r}$ are the equivalent control terms. The equivalent control terms make the system move along the sliding manifold under ideal conditions, and these terms can speed up the response of the system and reduce the steady-state errors.

The equivalent control terms are derived by letting $\dot{S}_{P}=\dot{S}_{Q}=0$. The power errors can be defined as below

$$
\left\{\begin{array}{l}
\dot{e}_{p}=\frac{-3}{2 L_{f}}\left[\left(U_{\alpha}^{2}+U_{\beta}^{2}\right)-\left(U_{\alpha} V_{\beta}+U_{\beta} V_{\alpha}\right)\right] \\
-\left(\frac{R_{f}}{L_{f}}\right) P-w Q-\dot{P}^{*} \\
\dot{e}_{Q}=\frac{3}{2 L_{f}}\left[\left(U_{\beta} V_{\alpha}+U_{\alpha} V_{\beta}\right)\right]-\left(\frac{R_{f}}{L_{f}}\right) Q-w P-Q^{*}
\end{array}\right.
$$

If we define the functions $H_{p}$ and $G_{Q}$ as follows:

$$
\left\{\begin{array}{l}
H_{p}=\frac{-3}{2 L_{f}}\left[\left(U_{\alpha}^{2}+U_{\beta}^{2}\right)-\left(U_{\alpha} V_{\beta}\right)\right]-w Q-\dot{P}^{*} \\
H_{Q}=\frac{3}{2 L_{f}}\left[\left(U_{\beta} V_{\alpha}\right)\right]-w P-Q^{*}
\end{array}\right.
$$

Then we have, the second derivate of the errors as

$$
\left\{\begin{array}{l}
\ddot{e}_{p g}=\frac{3}{2 L_{f}} U_{\beta} V_{\alpha}-H_{p g}-\left(\frac{R_{f}}{L_{f}}\right) \dot{P} \\
\ddot{e}_{Q g}=\frac{3}{2 L_{f}} U_{\alpha} V_{\beta}+H_{Q g}-\left(\frac{R_{f}}{L_{f}}\right) \dot{Q}
\end{array}\right.
$$

The indices $\mathrm{P}$ and $\mathrm{Q}$ being the active and reactive powers indices respectively

In finite time, based on the STA approach, the terms $V_{i S T}$ are calculated according to [11]

$\left\{\begin{array}{l}V_{P S T}=-\lambda_{p g}\left|e_{P g}\right|^{0.5} \operatorname{Sgn}\left(e_{P g}\right)-\chi_{p g} \int \operatorname{Sng}\left(e_{P g}\right) d t \\ V_{Q S T}=-\lambda_{Q g}\left|e_{Q g}\right|^{0.5} \operatorname{Sgn}\left(e_{Q g}\right)-\chi_{Q g} \int \operatorname{Sng}\left(e_{Q g}\right) d t\end{array}\right.$

Of which only the bounds $\Gamma_{m}, \Gamma_{M}$ and $\Phi$ are known:

$$
\left\{\begin{array}{l}
0<\Gamma_{m p g}<\frac{3}{2 L_{f}} U_{\beta}<\Gamma_{M p g},\left|H_{P g}-\left(\frac{R_{f}}{L_{f}}\right) \dot{P}\right|<\Phi_{p g} \\
0<\Gamma_{m Q g}<\frac{3}{2 L_{f}} U_{\alpha}<\Gamma_{M Q g},\left|H_{Q g}-\left(\frac{R_{f}}{L_{f}}\right) \dot{Q}\right|<\Phi_{Q g}
\end{array}\right.
$$

The sufficient conditions for finite-time convergence are,

$$
\begin{cases}\lambda_{p g}>\frac{\Phi_{p g}}{\Gamma_{M P g}}, & \chi_{p g}{ }^{2} \geq \frac{4 \Phi_{p g}\left(\lambda_{p g}+\Phi_{p g}\right)}{\Gamma_{m P g}{ }^{2}\left(\lambda_{p g}-\Phi_{p g}\right)} \\ \lambda_{Q g}>\frac{\Phi_{Q g}}{\Gamma_{M Q g}}, & \chi_{Q g}{ }^{2} \geq \frac{4 \Phi_{Q}\left(\lambda_{Q g}+\Phi_{Q g}\right)}{\Gamma_{m Q g}{ }^{2}\left(\lambda_{Q g}-\Phi_{Q g}\right)}\end{cases}
$$

This ensures that uncertainty on $L_{f}$ and $R_{f}$ will not be a threat to the robustness of the algorithm. However, accurate value of the $V_{i e q}$ control terms in (19) will greatly contribute to lower the control effort done by the STA.

In practice, the parameters are never assigned according to inequalities. Usually, the real system is not exactly known, the model itself is not really adequate, and the parameters estimations are much larger than the actual values. The larger the controller parameters, the more sensitive the controller to any switching measurement noises. The right way is to adjust the controller parameters during computer simulations.

The value of $k_{\text {sngi }} \operatorname{sign}\left(S_{i}\right)$ is determined by the fuzzy structure detailed in the section follow.

\section{FuZZY CONTROLLER}

Fuzzy-logic control has the capability to control nonlinear, uncertain and adaptive systems with parameter variation. Fuzzy control does not strictly need any mathematical model of the plant. 
Its control rule can be qualitatively expressed on the basis of logic-language variation and the fuzzy model of a plant is very easy to apply. In fact, fuzzy control is good adaptive control among the techniques discussed so far. In this paper, fuzzy-logic control is associated with sliding-mode control to generate the value of the disconnect component gain which ensures the precision and robustness of the control [12].

The general structure of a fuzzy-control system is shown in Fig. 3. There are two input signals to the fuzzy controller, the error $\mathrm{E}$ and the change in error $\mathrm{CE}$, which is related to the derivative $\mathrm{DE} / \mathrm{dt}$ of error. The closed-loop error $\mathrm{E}$ and change in error $\mathrm{CE}$ signals are converted to the respective scale factors, $\mathrm{e}=\mathrm{E} / \mathrm{GE}$ and $\mathrm{ce}=\mathrm{CE} / \mathrm{GC}$. The output plant control signal DU is derived by multiplying the per unit by the scale factor $\mathrm{GU}$, that is $\mathrm{DU}=\mathrm{du} * \mathrm{GU}$, and then integrated to generate the U signal [13].

The scale factors can change the sensitivity of the controller without changing its structure. The fuzzy controller is composed of three blocks: fuzzification, rule bases, and defuzzification. The function of membership of each input signal (E, dE) is illustrated in Fig. 4. The fuzzy subsets are as follows: NB (Negative Big), Nm (Negative Medium), NS (Negative Small), Z (Zero), PS (Positive Small), PM (Positive Medium), PB (Positive Big). There are seven fuzzy subsets for each variable, which gives $7 * 7=49$ possible rules, where

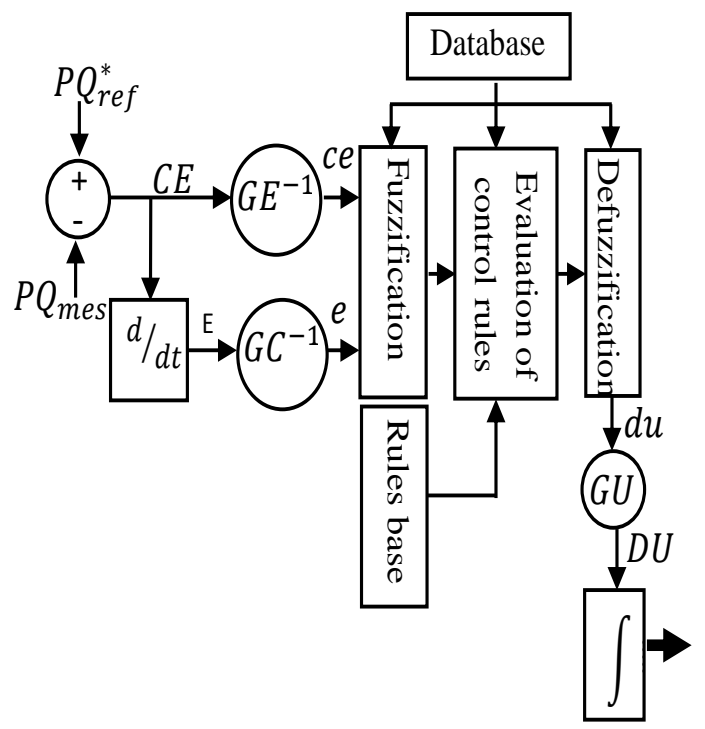

Fig. 3. Structure of the Fuzzy Controller

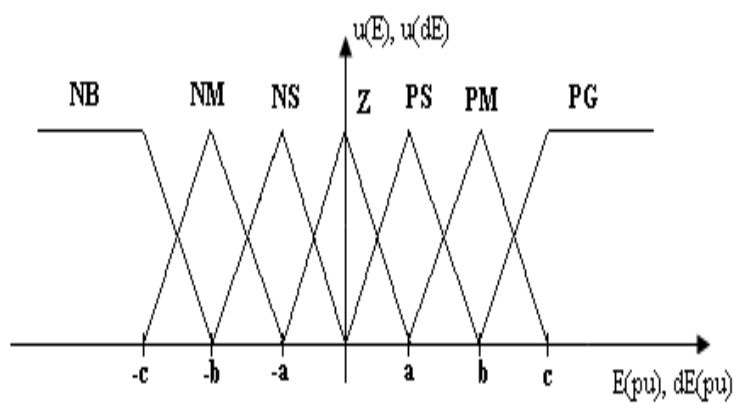

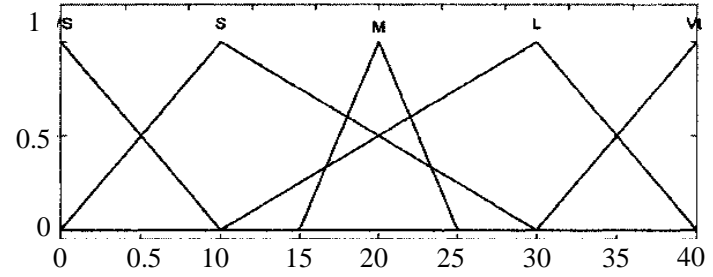

Fig. 4. b. Membership functions

TABLE I. RULES BASE

\begin{tabular}{|l|l|l|l|l|l|l|l|}
\hline & NB & NM & NS & $\mathbf{Z}$ & PS & PM & PB \\
\hline PB & Z & PS & PM & PB & PB & PB & PB \\
\hline PM & NS & Z & PS & PM & PB & PB & PB \\
\hline PS & NM & NS & Z & PS & PM & PB & PB \\
\hline $\mathbf{Z}$ & NB & NM & NS & Z & PS & PM & PB \\
\hline NS & NB & NB & NM & NS & Z & PS & PM \\
\hline NM & NB & NB & NB & NM & NS & Z & PS \\
\hline NB & NB & NB & NB & NB & NM & NS & Z \\
\hline
\end{tabular}

typical rule is: "If $\mathrm{E}(\mathrm{pu})$ is $\mathrm{PS}$ and $\mathrm{dE}(\mathrm{pu})$ is $\mathrm{PM}$, then $\mathrm{dU}$ (pu) PB." (Table.1). Defuzzification is done by the centroid method based on the Takagi-Sugeno-Kang inference method.

\section{Space Vector PwM}

We use a Model of three-phase, tow-level source SVPWM inverter with center-taped grounded DC bus. The relationship between the switching variable vector and voltage vector can be expressed below:

$$
V_{\alpha \beta}=\left[\begin{array}{c}
V_{\alpha} \\
V_{\beta}
\end{array}\right]=C 32\left[\begin{array}{c}
V_{a n} \\
V_{b n} \\
V_{c n}
\end{array}\right]=\frac{1}{3}\left[\begin{array}{lrr}
2 & -1 & -1 \\
-1 & 2 & -1 \\
-1 & -1 & 2
\end{array}\right]\left[\begin{array}{c}
C_{a} \\
C_{b} \\
C_{c}
\end{array}\right] \frac{V_{d c}}{2}
$$

Where $C_{32}$ is the Park transformation Matrix, $C_{a}, C_{b}$ and $C_{c}$ are the states of the interrupters.

Nabae and All in [14] divide the space vector plane into six sectors as shown in Fig.5. in the space vector approach, the desired reference vector is generated by time averaging the suitable discrete vltage vectors in sampling period $T_{s}$. For a given reference voltage Uref and angle in sector $\mathrm{n}$, the volttime balance is maintained by applying the active vectors 1,2 and zero states together for durations $T_{1}, T_{2}$ and $T_{0}$ respectively, as given in following expressions :

$$
\begin{gathered}
\int_{0}^{T_{z}} U_{r e f}=\int_{0}^{T_{1}} V_{1}+\int_{T_{1}}^{T_{1}+T_{2}} V_{2}+\int_{T_{1}+T_{2}}^{T_{0}} V_{0} \\
T_{1}=\frac{\sqrt{3} T_{z}\left|U_{r e f}\right|}{V_{d c}}\left(\sin \left(\frac{n}{3} \pi\right) \cos (\alpha)-\cos \left(\frac{n}{3}\right) \sin (\alpha)\right)
\end{gathered}
$$

Fig. 4. a. Member functions 


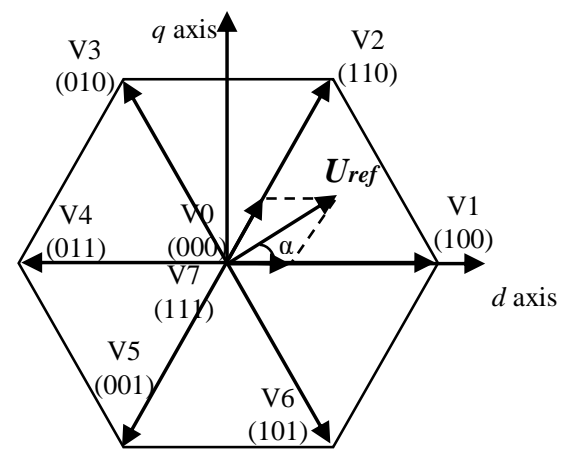

Fig. 5. Basic switching vectors and sectors

$$
\begin{gathered}
T_{2}=\frac{\sqrt{3} T_{z}\left|U_{r e f}\right|}{V_{d c}}\left(\sin (\alpha) \cos \left(\frac{n-1}{3} \pi\right)-\cos (\alpha) \sin \left(\frac{n-1}{3} \pi\right)\right) \\
T_{0}=T_{z}-\left(T_{1}+T_{2}\right)
\end{gathered}
$$

With $\quad\left(T_{z}=\frac{1}{f_{z}}\right)$

Where: $\mathrm{n}=1$ through 6 (that is, Sector 1 to 6 ) and $0<\alpha<60^{\circ}$

\section{SIMULATION RESULTS}

The power generated by the wind system is transmitted to the grid via the stator of the DFIG (Fig.1). On the other hand, only the slip power is exchanged between the grid and the rotor of the DFIG. When the DC link voltage is well regulated, the rotor current should be ensuring the smooth exchange of slip power.

Simulations have been carried out to demonstrate the effectiveness of proposed control strategy applied to the grid connected converters of a wind energy system. The proposed design is implemented in Matlab/Simulink software packages using parameters given in Table 2 .

Simulink discrete models with step time of $1 \mu$ s were used. A programmable 3-phase voltage source was used to simulate the grid with voltage fluctuations. The IGBTs of the GSC were driven by a SV-PWM generator.

The bloc diagram of Fig. 6 shows the proposed sliding mode power control for the GSC. An outer loop (not shown) contains PI controller to regulate the dc-link voltage and produce the active power reference. The control law developed in the previous section directly generates the converter voltage reference in the stationary $\alpha \beta$ frame, according to the instantaneous values of the errors on the active and reactive powers.

The system is simulated for two different cases: first insteady state, in order to demonstrate the DC link voltage regulation and power factor control. In the second case, a transient state with system parameters variation was simulated. The objective of this second simulation is to prove the robustness of proposed strategy.

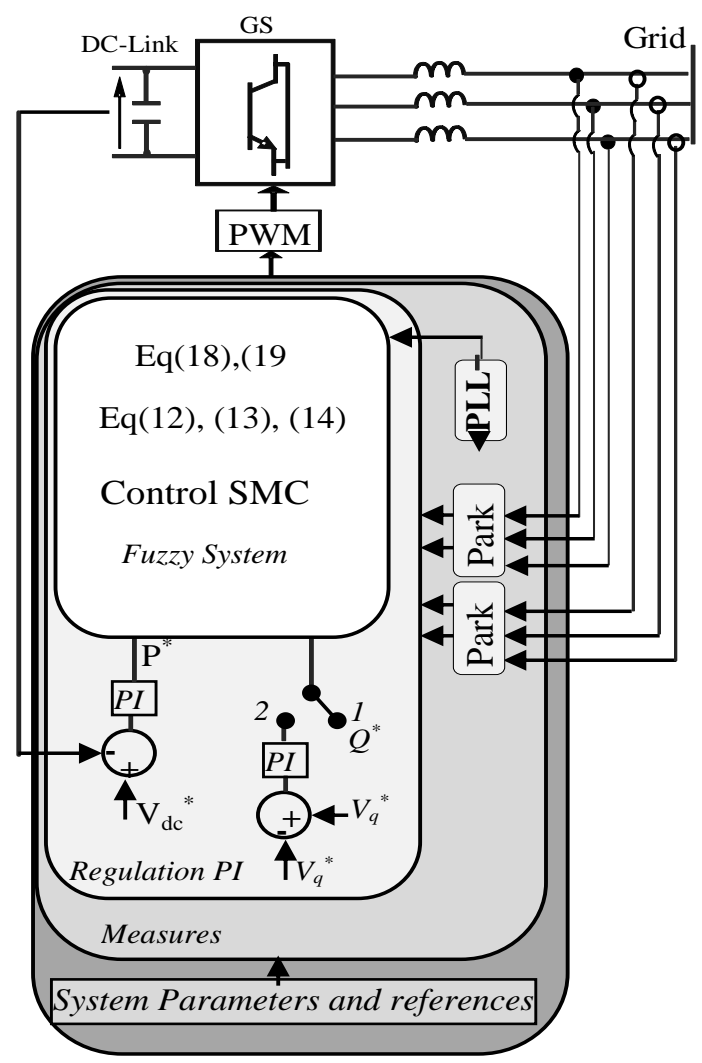

Fig. 6. Bloc diagram of WECS connected to the grid

Case1:

In this case, the voltage of the programmable source is fixed to constant frequency and magnitude.

The RSC and DFIG rotor are simulated by a constant current source and a well smoothed wind power is assumed. Imposing $\mathrm{Q}=0$, an initial current of $\mathrm{Is}=1.5 \mathrm{~A}$ is supplied to the DC-link circuit in order to simulate a slip power extracted from the rotor. At $\mathrm{t}=0.3 \mathrm{~s}$, a current step is induced, bringing its value to $-1.5 \mathrm{~A}$ (Fig.7). This is done to demonstrate the system's ability to transmit and received power to/from the grid. At $t=0.6 \mathrm{~s}$ the current is brought back to its initial value of $1.5 \mathrm{~A}$. The results presented on Fig. 8 and 10 show that both the DC- link voltage and reactive power are unaffected by the dc current steps. Fig.9 shows the active power dynamics, starting at $-600 \mathrm{~W}$ and reaching the value of $+600 \mathrm{~W}$ on the first step at $\mathrm{t}=0.3 \mathrm{~s}$, and then going back to the initial value of $-600 \mathrm{~W}$ at $0.6 \mathrm{~s}$. This behavior corresponds to the transition from hypo- to hyper-synchronous operating modes of the DFIG. The power involved, which is transported by the

TABLE II. PARAMETERS OF THE TESTED GSC SySTEM

\begin{tabular}{|l|l|l|l|}
\hline Rated power & $1.2 \mathrm{~kW}$ & Line resistance & $0.001 \mathrm{H}$ \\
\hline Grid rated voltage & $208 \mathrm{~V}$ & Line inductance & $0.001 \Omega$ \\
\hline Grid rated frequency & $60 \mathrm{~Hz}$ & DC link voltage & $400 \mathrm{~V}$ \\
\hline DC link capacitor & $35000 \mu \mathrm{F}$ & \multicolumn{2}{|c}{} \\
\cline { 1 - 2 } & &
\end{tabular}




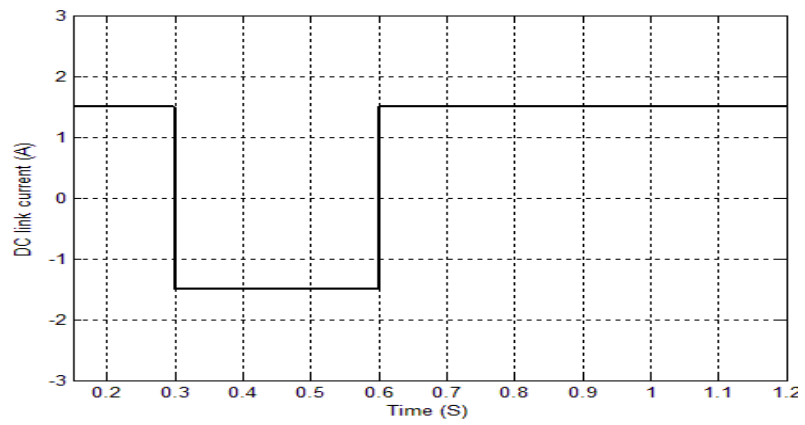

Fig. 7. DC link current

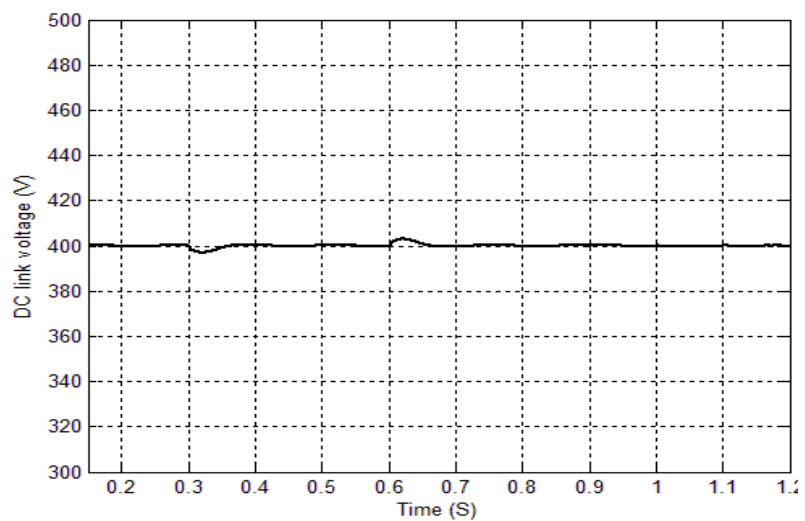

Fig. 8. DC link voltage

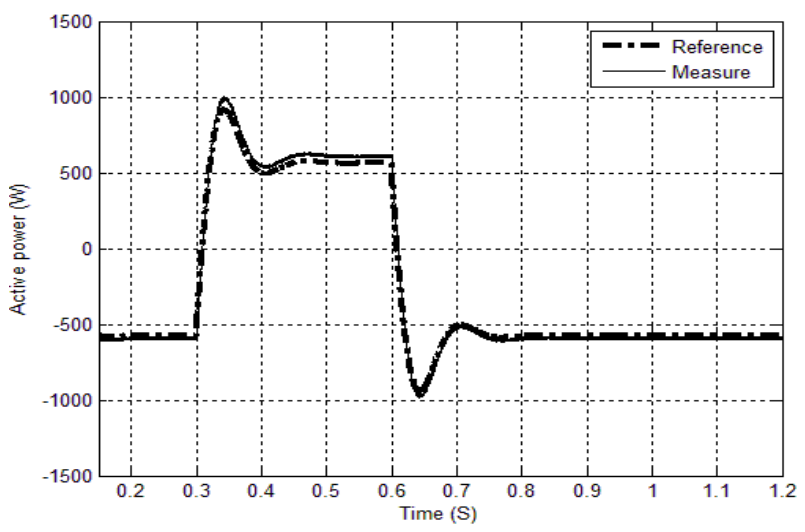

Fig. 9. Active power reference and response

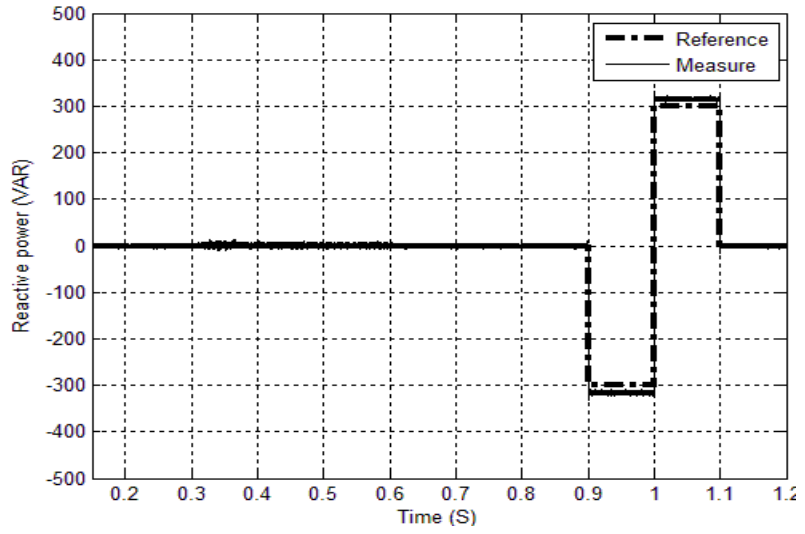

Fig. 10. Reactive power reference and response

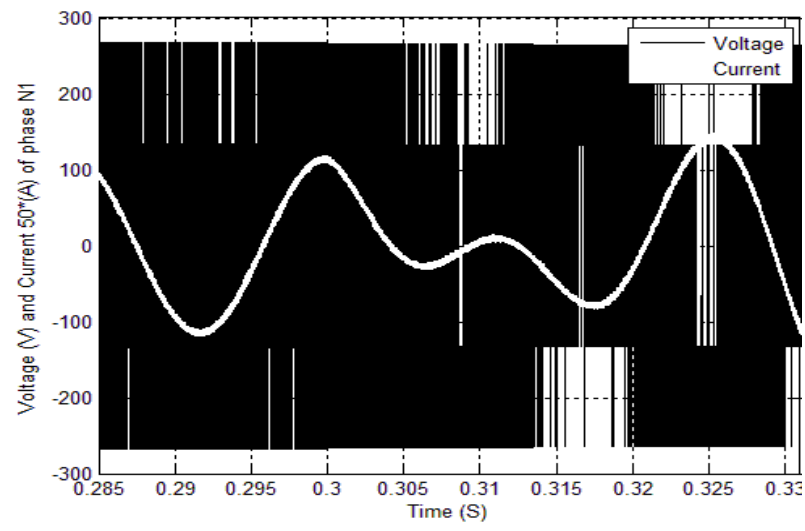

Fig. 11. Transition of current at first transition of the DC current

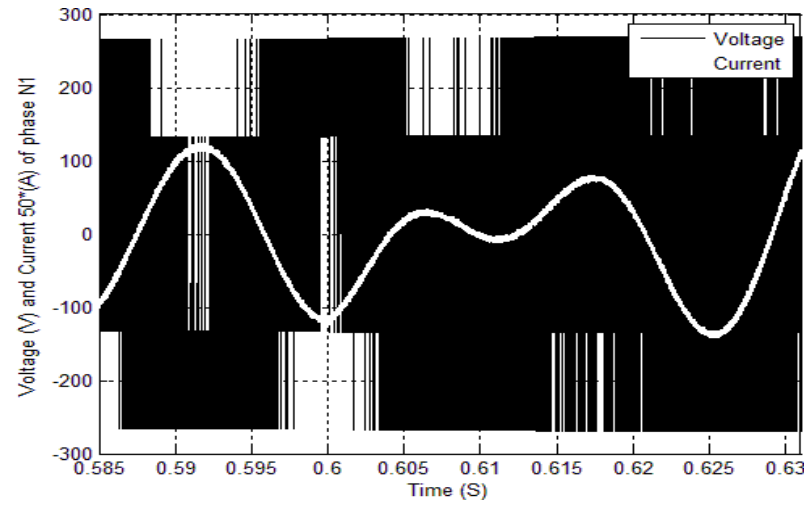

Fig. 12. Transition of current at second transition of the DC current

GSC, is $50 \%$ of the GSC nominal power. The passage between hyper- and hypo-synchronous modes is made without great transient current, which give attenuation at the power transient state. An overshoot in power peaking to twice the permanent value is considered reasonable.

In order to demonstrate the decoupling between active and reactive powers, the latter is varied by steps from $0.9 \mathrm{~s}$ to 1.1 $\mathrm{s}$. At $\mathrm{t}=0.9 \mathrm{~s}$ a step brings $\mathrm{Q}$ from 0 to -300 VAR then to $+300 \mathrm{VAR}$ at $\mathrm{t}=1.0 \mathrm{~s}$ and finally back to $0 \mathrm{VAR}$ at $\mathrm{t}=1.1 \mathrm{~s}$. (Fig.10)

As can be seen the step change of one control variable, i.e. active or reactive power, does not affect the other, and there is no high overshoot of the active and reactive powers. Fig.11 and Fig.12 show that the initial power factor of 1 is changed to -1 after the first step in the current Is, and then changed back from -1 to 1 .

Case 2:

For this case, a perturbation is introduced under the form of a short circuit defect that causes a symmetrical voltage amplitude dip on the grid.

On the simulated model, the programmable voltage source is set to change its magnitude at $\mathrm{t}=0.4 \mathrm{~s}, 0.7 \mathrm{~s}$ and $1 \mathrm{~s}$, in order to demonstrate the ability of the designed control scheme to maintain the system performances. The corresponding voltage magnitude passes from nominal value $\mathrm{Vn}$ to $0.7 \mathrm{Vn}$, then to $1.3 \mathrm{Vn}$, and finally back to the nominal value (Fig.13). 


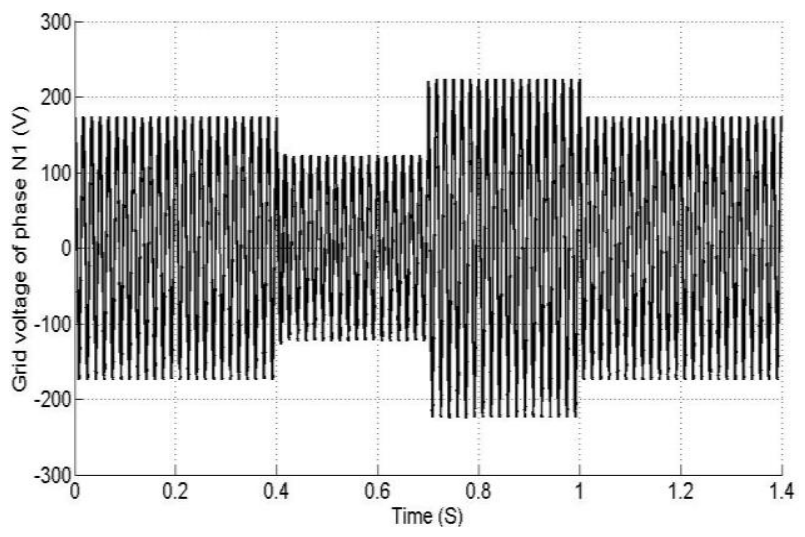

Fig. 13. Grid voltage at transient state

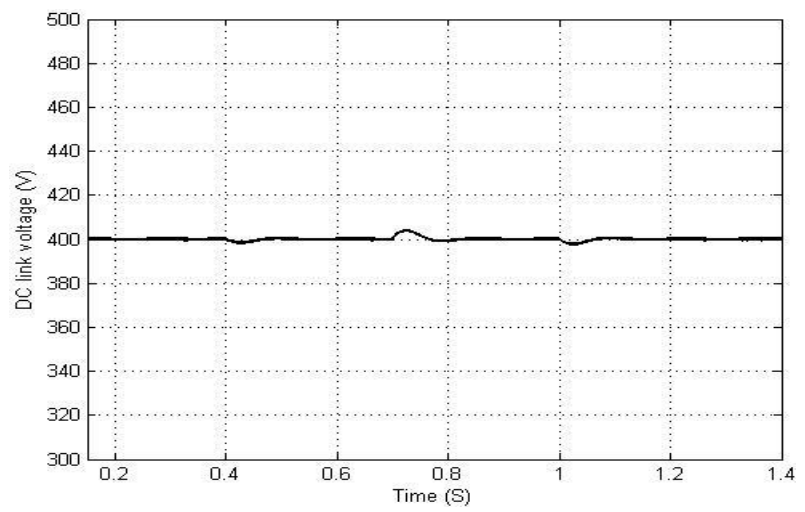

Fig. 14. DC link voltage at transient state

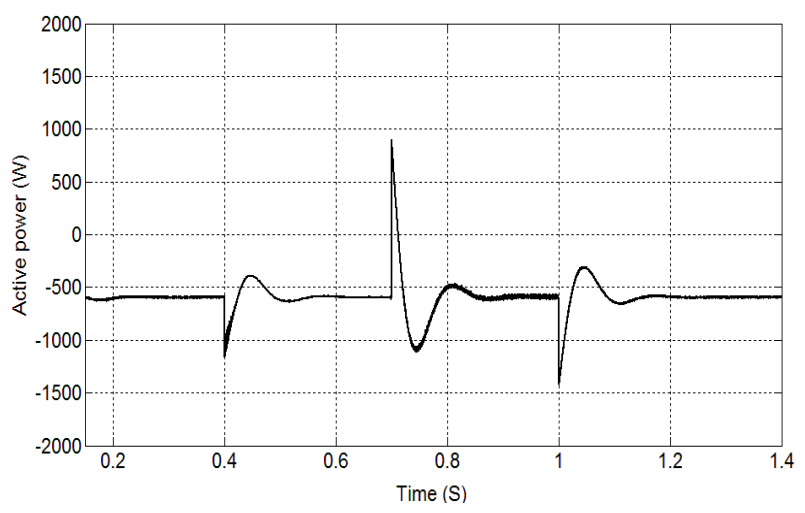

Fig. 15. Active power at transient state

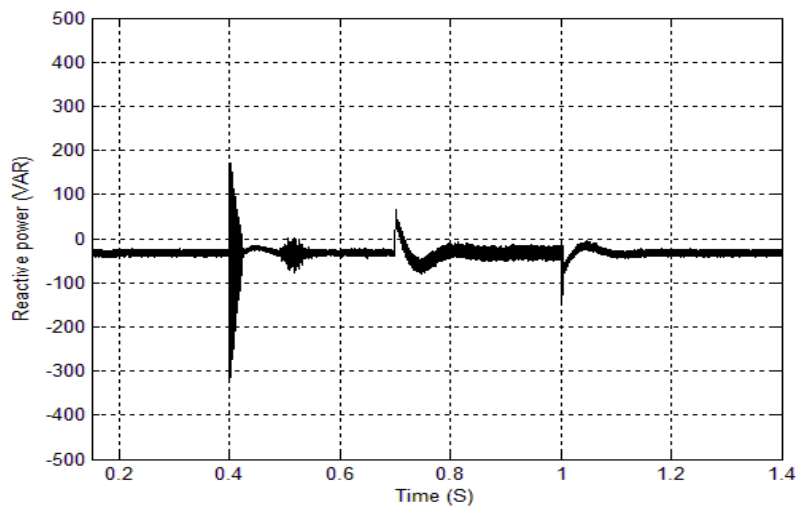

Fig. 16. Reactive power at transient state

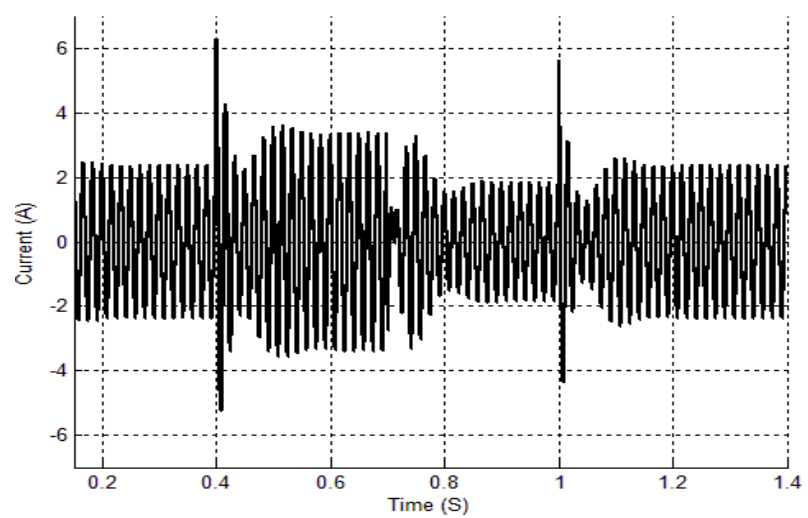

Fig. 17. Grid current at transient state

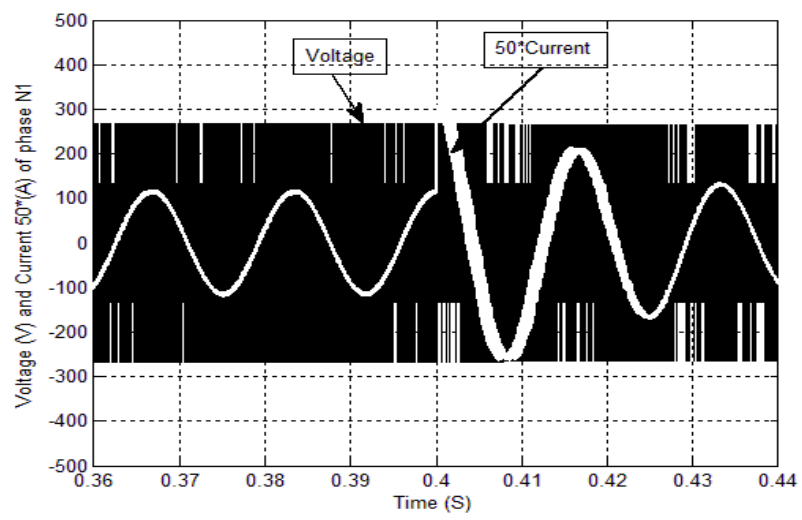

Fig. 18. Grid current and voltage at first transition of voltage

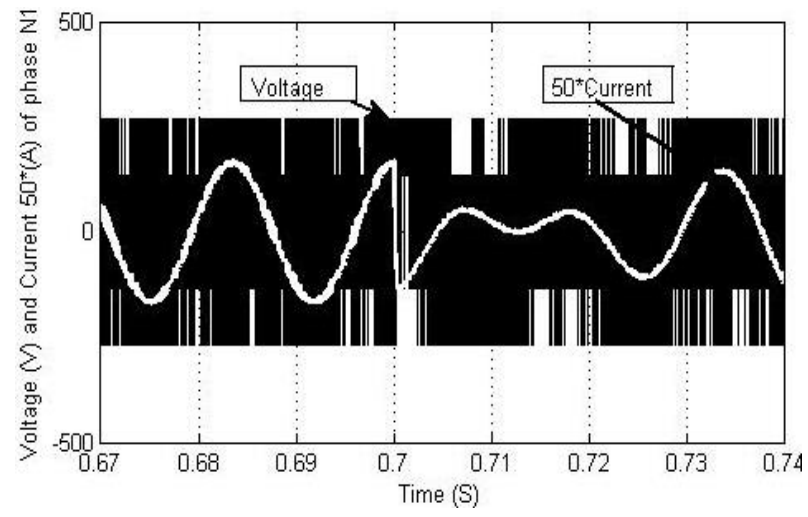

Fig. 19. Grid current and voltage at second transition of voltage

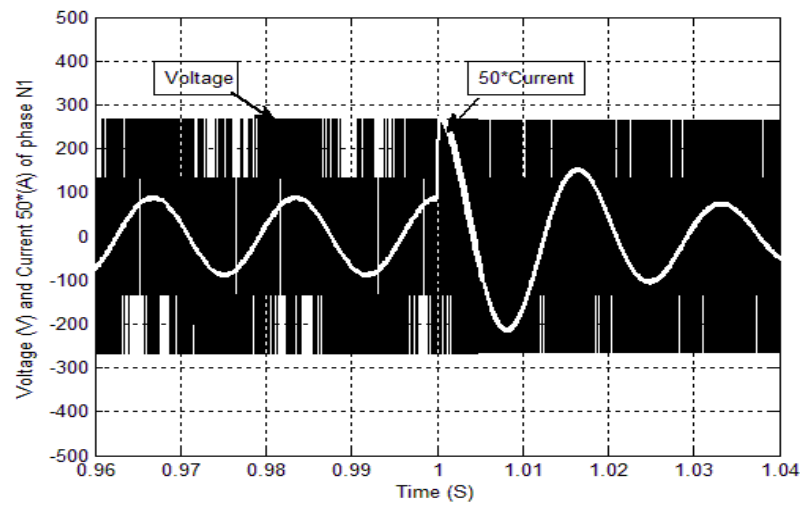

Fig. 20. Grid current and voltage at third transition of voltage 
In addition to the voltage variations, the values of inductance and resistance were changed to $50 \%$ of their value. The objective in that is to demonstrate the control scheme robustness towards both perturbations: grid fault and parameters identification.

Fig.14 to Fig.20 presents the dynamic behavior of the system for this case. In Fig.14 the DC link voltage appears roughly constant for all perturbations.

Fig.15 shows a more violent transient on the active power compared to the Fig. 9 in for the first case. At $t=0.7 \mathrm{~s}$, the peak reach $800 \mathrm{~W}$ which represents a variation of nearly three times the permanent value $(-600)$. Meanwhile, the reactive power shown on Fig. 16 reaches -300 VAR at $t=0.4 \mathrm{~s}$. This value represents 15 times the permanent value of -20 VAR. However, this peak takes just a few milliseconds, which makes it negligible.

The current also reaches peak values during transient states of few milliseconds (Fig.17). These peaks are predicable due to the voltage disturbance. In all transient states, the current gets back to a steady value after $200 \mathrm{~ms}$ approximately, which we think is acceptable considering there are not high transient peak values. Zooms on the current are helpful to see the behavior of the current and voltage during the first transient state at $0.4 \mathrm{~s}$ (Fig.18), during second transient state at $0.7 \mathrm{~s}$ (Fig.19) and finally during third transient state at 1s (Fig.20).

\section{CONCLUSION}

A SOSM control scheme for independent control of active and reactive power of a VSC (RSC) has been presented. The proposed SM-DPC scheme directly generate the SV-PWM switching pattern that forces the close loop system to slide along a desired state trajectory, designed to minimize the active and reactive power errors. The control law has also been formulated to ensure stability and alleviate the chattering effect.

To demonstrate the feasibility of the proposed fuzzySOSM strategy, two simulation cases were considered: constant and faulted (variable) grid voltage conditions. The simulations results obtained demonstrate the robustness and the good performances in terms of stability and tracking of the reference state trajectory. The pretty well features of the proposed fuzzy SOSM based DPC strategy are as follows

1) No rotating coordinate transformation and angular information of grid voltage are required.

2) Enhanced transient performance

3) The steady-state and transient responses are insensitive to the system parameter's variations.
4) Chattering-free behavior, a finite reaching time, and robustness with respect to external disturbances (grid)

The contribution of the proposed control to enhance system performances and experimental validation will be the subjects of a coming paper.

\section{REFERENCES}

[1] J. M. Carrasco, L. G. Franquelo, J. T. Bialasiewicz, E. Galvan, R. C. P. Guisado, M. A. M. Prats, J. I. Leon, and N. Moreno-Alfonso, "Powerelectronic systems for the grid integration of renewable energy sources:

a survey," IEEE Trans. Ind. Electron., vol. 53, no. 4, pp. 1002-1016,

Aug. 2006.

[2] S. Janardhanan,, and B. Bandyopadhyay, "Output Feedback SlidingControl for Uncertain Systems Using Fast Output Sampling Technique," IEEE Transactions on Industrial Electronics, Vol. 53, $\mathrm{N}^{\circ} .5$, October 2006.

[3] T. C. Kuo, Y. J. Huang, C. Y. Chen, and C. H. Chang, "Adaptive Sliding Mode Control with PID Tuning for Uncertain Systems," Engineering Letters, Vol.16 N³, EL_16_3_06, August 2008.

[4] V. I. Utkin, J. G"uldner, and J. X. Shi, "Sliding Mode Control in Electromechanical Systems," FL: CRC Press, Boca Raton, 1999, pp. $115-130$

[5] Slotin, J.J. and Li,W., Applied non-linear control, Prentice Hall 1991.

[6] V. I. Utkin, "Sliding mode control design principles and applications to electric drives," IEEE Trans. Ind. Electron., vol. 40, no. 1, pp. 23-36,

Feb. 1993.

[7] O.A. Morfin, A.G. Loukianov, R. Ruiz, E.N. Sanchez, F.Valenzuela, M. I. Castellanos, "Grid side converter controller applied in wind systems via second order sliding modes," 8th International Conference on Electrical Engineering Computing Science and Automatic Control (CCE), Mexico, 2011, pp.1-6.

[8] Yao.08 Yao, Chuanbao Yi, Deng ying, Jiasi Guo and Lina Yang,"The Grid-side PWM Converter of the Wind Power Generation System Based on Fuzzy Sliding Mode Control," International Conference on Advanced Intelligent Mechatronics, Xiang, China,2008, pp.973-978.

[9] D. Kairous, R. Wamkeue, "Sliding-mode control approach for direct power control of WECS based DFIG,"EEEIC, Italy, 8-11 May 2011. Pp. 1-4, 2011.

[10] B. J. Parvat, B. M. Patre, "Second Order Sliding Mode Controller for Second Order Process with Delay Time," International Conference on Industrial Instrumentation and Control (ICIC), India. May 28-30, 2015. Pp 280-284.

[11] L. Fridman, and A Levant, "Higher order sliding modes as a natural phenomenon in control theory," Ser. Lectures Notes in Control and Information Science, F. Garafalo, and L. Glielmo, Eds. New York, Springer-Verlag, 1995, Vol. 217, pp. 107-133.

[12] Y. Ren, H. Li, J. Zhou, Z. An, J. Liu, H. Hu, and H. Liu, "Dynamic Performance Analysis of Grid-Connected DFIG Based on Fuzzy Logic Control," ICMA, August 9-12, China 2009.

[13] L. A. Zadeh, "Fuzzy Setes,"Information and Control, vol. 8, pp.338-353, 1965.

[14] Nabae A., Ogasawara S., Akagi H., A novel control scheme for current controlled PWM inverters, IEEE Trans. Ind. Applicat., 22 (1986), No. 4, 312-323. 